\title{
Design and Implementation of Remote Control System for Smart Home PTZ Based on WEB
}

\author{
Zhigang Ren ${ }^{1}$, Hao $\mathrm{Liu}^{2, *}$, Qiang $\mathrm{Li}^{3}$ \\ Department of Information Engineering of Beijing Institute of Fashion Technology \\ Beijing , China
}

891862512@qq.com,gxyliuh@bift.edu.cn,976065576@qq.com

\begin{abstract}
This paper introduces the design of the remote web-based control system and the implementation of software and hardware under the embedded Linux operating system, and studies the implementation of dynamic web technology. The design method of CGI program realizes the remote control system using BOA as the web server under the embedded Linux platform, and successfully realizes the remote control of the rotation of the two-dimensional servo in the up, down, left and right directions.
\end{abstract}

Keywords— WEB ; CGI ; BOA ,two-dimensional steering gear

\section{OVERVIEW}

The web-based remote control system is based on the tiny6410 development board hardware platform and the embedded linux operating system. The embedded web server is used to control the stepping motor. The system is divided into two parts: the client host and the on-site embedded web server control device. The entire remote control system uses the $\mathrm{B} / \mathrm{S}$ architecture, and the remote control terminal, the client browser, can send data to the web server through the network $^{[1]}$. The embedded control system builds a web server on the development board, accept and sent the data by the client browser, and then controls the rotation of Steering gear through the embedded linux system. The system uses boa as the embedded web server, which is responsible for receiving and analyzing user requests, transmitting static pages and calling back-end CGI programs.

\section{SYSTEM DESIGN AND IMPLEMENTATION}

With the development of society, people's requirements for the safety of the living environment are getting higher and higher. The ordinary safety gates and security systems have not met the safety requirements of people. Due to the development of Internet of things technology, smart home has also developed rapidly. The smart home cloud platform remote control system enables people to monitor the security

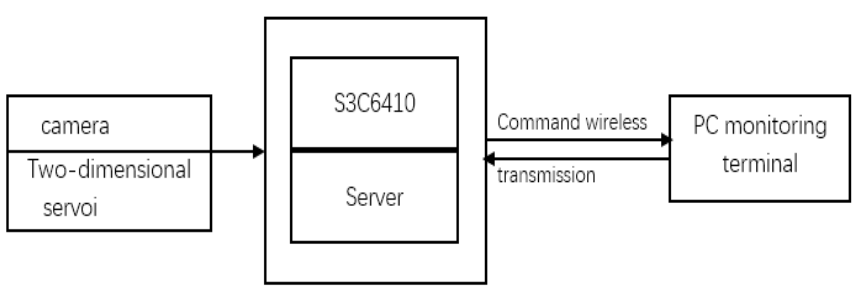

situation around the home. The whole system is divided into cloud head, PC monitoring terminal and server side. In the PC side, the webpage is manually monitored and the cloud is manually changed. In the direction of the platform, the system uses a two-dimensional steering gear to control the direction of the gimbal. The system framework is shown in Fig.1.

Fig.1. System framework

\section{SYSTEM HARDWARE}

A. System hardware platform 
The processor is the core of the system hardware. Due to the low power consumption and low cost of ARM, the system uses ARM11 (S3C6410) as the main control chip and Tiny6410SDK as the core board of the main processor. The S3C6410 is based on the ARM1176JZF-S core. It is a high performance 32-bit RISC microprocessor with a clock speed of up to $667 \mathrm{MHz}$. It uses a 64/32-bit internal bus architecture and integrates a powerful multimedia processing unit to

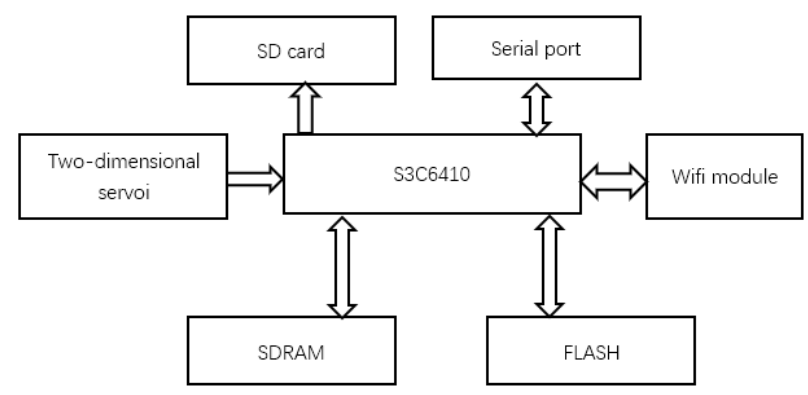

support Mpeg4, H. The video file hardware codec of 264/H.263 format can be output to the LCD display; the core board integrates $128 \mathrm{M} / 256 \mathrm{M}$ DDR RAM, SLC NAND Flash $(256 \mathrm{M} / 1 \mathrm{~GB})$ or MLC NAND Flash (2GB) memory, and is powered by $5 \mathrm{~V}$. The board implements various core voltage conversions necessary for the CPU, and also has a reset chip, three LCD interfaces, a 4-wire resistive touch screen interface, a $100 \mathrm{M}$ standard network interface, a standard DB9 five-wire serial port, a Mini USB 2.0 interface, and a USB Host 1.1. $3.5 \mathrm{~mm}$ audio input and output port, standard TV-OUT interface, SD card holder, Mini PCIe interface, capacitive screen interface, etc, provide powerful hardware support for secondary development, system hardware architecture shown in Fig. 2.

Fig. 2. System hardware architecture

\section{B. Working principle of the steering gear}

The control signal enters the signal modulation chip from the channel of the receiver to obtain a DC bias voltage. It has a reference circuit inside, which generates a reference signal with a period of $20 \mathrm{~ms}$ and a width of $1.5 \mathrm{~ms}$, and compares the obtained DC bias voltage with the voltage of the potentiometer to obtain a voltage difference output. Finally, the positive and negative voltage output of the voltage difference to the motor drive chip determines the forward and reverse of the motor. When the motor speed is constant, the potentiometer is rotated by the cascade reduction gear, so that the voltage difference is 0 , and the motor stops rotating. The control of the steering gear generally requires a time base pulse of about $20 \mathrm{~ms}$. The high level portion of the pulse is generally an angle control pulse portion in the range of $0.5 \mathrm{~ms}$ to $2.5 \mathrm{~ms}$, with a total interval of $2 \mathrm{~ms}$. The steering angle of the system servo is 180 degrees, then the corresponding control relationship is as follows:

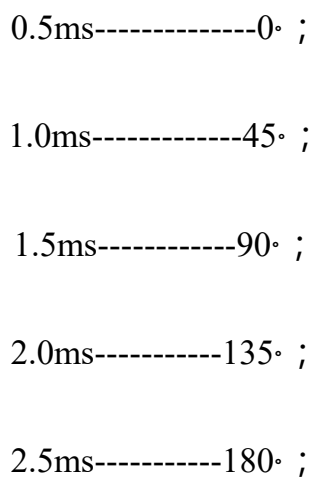

Follow-up characteristics of the steering gear: Assume that the steering gear is now stable at point A. At this time, the CPU sends a PWM signal, and the servo is turned from point A to point B at full speed. In this process, it takes a while for the servo to move to point B. Hold time is Tw.

When $T w \geq \Delta T$, the servo can reach the target and have the remaining time;

When $\mathrm{Tw} \leq \Delta \mathrm{T}$, the steering gear cannot reach the target; Theoretically: when $\mathrm{Tw}=\Delta \mathrm{T}$, the system is the most consistent, and the steering gear is the fastest.

\section{SYSTEM SOFTWARE DESIGN}

The system uses a web page to manual control the steering gear on online, and the browser requests the URL of a CGI application through an HTML form ${ }^{[1]}$. The server received the request, then the server executes the specified CGI application. The CGI application executes the servo driver to indirectly control the rotation direction of the gimbal. The System software working relationship is shown in Fig. 3.

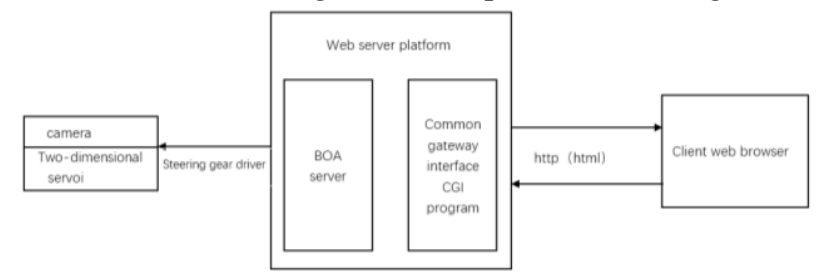


Fig.3. System software working relationship

\section{A. Introduction to CGI}

CGI is a Universal Gateway Interface. It is actually an application that runs on a WEB server and is triggered by user input from the browser to provide an interface to the client's HTML page. It allows network users to access and run applications on the host where the remote server system resides, further control the associated hardware, format the output into HTML format, and ultimately feed back to the browser. This completes the dynamic interaction between the HTML static page and the web server.

CGI is different from JAVA Web. The dynamic web page or web program developed by JAVA transmits the java application to the client through the network, and then the client runs the corresponding Java program, but CGI runs on the server. It is their biggest difference! Therefore, it is possible to choose a method to develop a dynamic web page according to one's own needs, and realize remote control of an embedded device in the embedded field, and the CGI way to dynamically interact with the web page becomes the preferred solution.

\section{A. Steering gear drive software design}

Two GPIO ports (GPF14 and GPF15) on the development platform used in the system can be set as PWM outputs to control the rotation direction of the two-dimensional servo. In the browser, set the buttons in four directions ${ }^{[3]}$. After the operation, the system starts the CGI program, judges the operation code, and then starts to output the corresponding PWM information to the GPIO port to achieve the purpose of controlling the servo.

\section{1) Steering gear driver design}

When using the servo, the driver of the servo is call ed back, so that the system outputs a signal to the target port. The hardware driver is the interface between the e xternal device and the operating system kernel. The drive $r$ hides the details of the hardware for the application, $s$

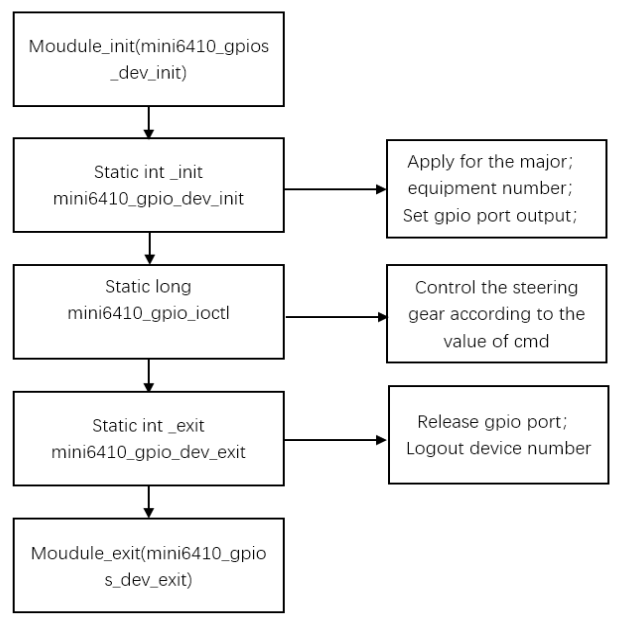

$\mathrm{o}$ for the application, the hardware device is equivalent $\mathrm{t}$ o a device file, and the application can operate with you $r$ device ${ }^{[4]}$. The servo drive module is a character type $d$ evice. The driver design of the servo is shown in Fig. 4. It is mainly divided into three parts, namely the initiali zation I/O port, and the GPIO main device number ret $=\mathrm{r}$ egister_chrdev(GPIO_MAJOR, DEVICE_NAME, \&mini64 10_gpio_fops ); according to the cmd command to chang e the movement of the servo and exit the device module. The driver is compiled to generate a ko file and then 1 oaded into the Linux kernel.

Fig. 4. Servo drive design flow

\section{2) CGI programming}

CGI is an application running on the server. In the browser, the user inputs an instruction to trigger. The workflow is shown in Fig. 5. The CGI program contains control commands that will be passed to the driver module. The driver module receives commands to control the steering wheel in the up, down, left, and right directions. The CGI program is written as shown. First, output the content in HTML format, which is convenient for feedback to the browser, and then obtain the environment variable. Extract the value of the required variable from the obtained environment variable, open the GPIO port of the development board, and control the servo according to the command input by the browser $^{[5]}$. Finally, turn off the servo.

Fig. 5. Servo CGI program design flow

\section{SYSTERM TEST}

Install the servo driver into the kernel, then download the linux kernel to the development board, start the server, and

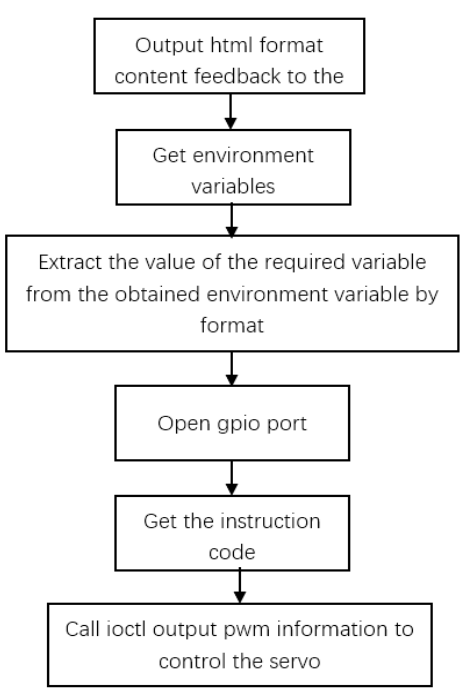


enter the development board address (http://192.168.1.230) in the web browser, as shown in Fig. 6 and Fig.7. Remotely control the camera orientation.

\section{ACKNOWLEDGEMENTS}

This work was supportted by the Post-Graduate Offcampus Talents Training Base(Project NO.120301990121/0 05).

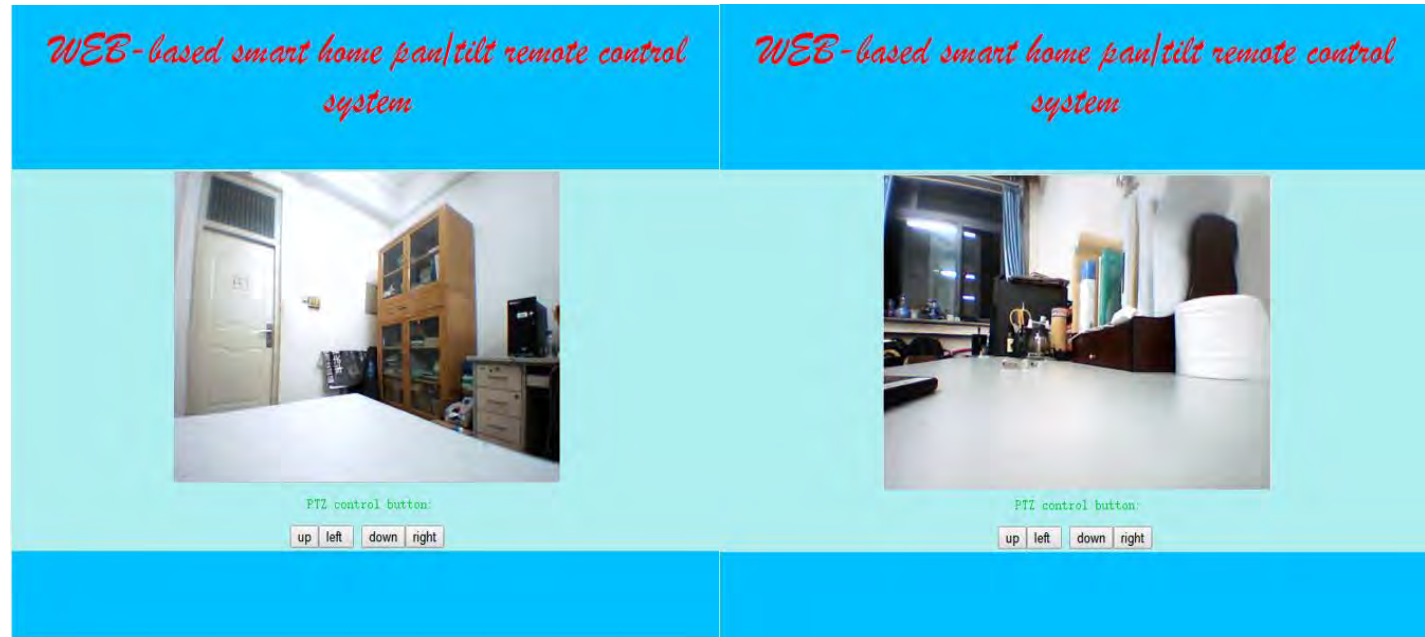

Fig. 6. Real-time monitoring 1

\section{CONCLUSION}

At this stage, the automatic supply of parts in industrial production, as well as robots, smart homes and other fields can be seen in the wide application of steering gear. The method based on embedded Web remote control system can realize the control of stepping motor, which can save a lot of money. The system's hardware and software resources, only the browser, no need to develop application software, low system cost. The monitoring platform is independent of the server. It is easy to expand new functions. System upgrade only needs to add corresponding modules on the web server side to reduce system upgrade and maintenance costs.
Fig. 7. Real-time monitoring 2 REFERENCE

[1] Wang Jingyuan. Research and design of embedded web system for remote control [J]. Software Development and Design 2011 (5).

[2] Li Beidou. Research on Design and Control of Digital Steering Gear [D]. Wuhan: China University of Geosciences, 2009.

[3] Li Xu, Xie Yunxiang. Overview of PWM Technology Implementation Methods [J]. Power Technology Application, 2005 (2): 51-55.

[4] Huo Lixia, Luo Weibing, Chi Xiaopeng. Design of multi-channel servo controller[J]. Modern Electronic Technology, 2010 (21): 73-75.

[5]JIANG Chenfei, LIU Zilong, HU Shaokai, et al. Research on control precision of multiple servos based on AVR single chip microcomputer[J].Information Technology, 2014 (3) : 